\title{
Zensus der Zukunft?
}

\section{Erdbeobachtung zur räumlichen Abschätzung der Bevölkerungsverteilung}

\author{
Michael Wurm • Hannes Taubenböck • Jan Goebel • \\ Thomas Esch · Gert G. Wagner
}

Online publiziert: 16 . November 2011

(C) Springer-Verlag 2011

Der Anteil der städtischen Bevölkerung an der Gesamtbevölkerung ist in industrialisierten Ländern traditionell hoch. Aber auch global gesehen wird der urbane Bevölkerungsanteil weiter steigen. Insbesondere in Entwicklungs- und Schwellenländern wird ein Großteil des prognostizierten Bevölkerungswachstums von Städten absorbiert werden. Rasant wachsende Städte - heutige und zukünftige Megacities - kommen mit der konventionellen statistischen Zählung der Bevölkerung und deren kontinuierlichem Anstieg oft nicht mehr nach. Was übrig bleibt sind grobe Schätzungen - oder die Nutzung von Erdbeobachtungsdaten. Auch Städte in Industrieländern stehen häufig vor dem Problem, dass keine genauen Aussagen über die aktuell ansässige Bevölkerung oder deren kleinräumige Verteilung getroffen werden kann. Satellitenbilder hingegen machen es möglich, große Gebiete der Erdoberfläche mit der nötigen Aktualität bei hoher geometrischer und thematischer Informationstiefe

Mag. rer. nat M. Wurm $(\bowtie) \cdot$ Dr. H. Taubenböck $\cdot$ Dr. T. Esch

Deutsches Zentrum für Luft- und Raumfahrt (DLR),

Deutsches Fernerkundungsdatenzentrum (DFD),

Münchner Straße 20, 82234 Weßling, Deutschland

E-Mail: michael.wurm@dlr.de

Dr. H. Taubenböck

E-Mail: hannes.taubenboeck@dlr.de

Dr. T. Esch

E-Mail: thomas.esch@dlr.de

Dr. J. Goebel

Längsschnittstudie Sozio-oekonomisches Panel, DIW Berlin, Mohrenstraße 58, 10117 Berlin, Deutschland

E-Mail: jgoebel@diw.de

Prof. Dr. G. G. Wagner

Deutsches Institut für Wirtschaftsforschung (DIW Berlin),

Mohrenstraße 58, 10117 Berlin, Deutschland

E-Mail: chairman@diw.de abzubilden. Mittels großmaßstäblicher Aufnahmen können selbst einzelne Häuser in Städten identifiziert werden, wodurch indirekt auf die Population - und ihre Verteilung im Raum - geschlossen werden kann.

\section{Bevölkerungsdynamik als Herausforderung}

$\mathrm{Ob}$ in Kairo heute 15 oder 25 Mio. Menschen leben, vermag niemand genau zu sagen. Die Megastadt ist geprägt von einem rapiden Bevölkerungswachstum inklusive aller damit einhergehenden Probleme: Slumbildung, unzureichende Versorgung, Verkehrskollaps und vieles mehr. Als größte Stadt der arabischen Welt zieht sie wie ein Magnet Menschen aus dem ganzen Land und den umliegenden Regionen an. In Kairo gibt es keine Meldepflicht, deshalb können Behörden nur von groben Schätzungen ausgehen.

Aus Sicht der industrialisierten Staaten erscheint die Frage nach der in einer Stadt wohnenden Bevölkerung bizarr. Doch auch für die Industrienationen Europas oder die Vereinigten Staaten Amerikas ist es durchaus schwierig, genaue Auskünfte über die aktuelle Bevölkerungszahl größerer Kommunen und insbesondere für einen gesamten Staat zu geben - sofern eine Zählung der Gesamtbevölkerung überhaupt durchführbar ist (Bradburn 1993). In den USA zum Beispiel gibt es kein einheitliches Personenregister, weshalb es für amerikanische Staatsbürger vor Wahlen auch notwendig ist, sich zu registrieren.

In Deutschland ist es Aufgabe des Statistischen Bundesamtes, die offiziellen Zahlen über Bevölkerungsgrößen auf Gemeindeebene zu berechnen und regelmäßig zu aktualisieren (Bundesministerium der Justiz 2009). Abhängig von der Größe und der administrativen Struktur kann jede Gemeinde zusätzlich ihre eigenen Aufzeichnungen - unter- 
teilt in untergeordnete Verwaltungseinheiten wie Stadtbezirke oder Stadtviertel - führen.

Auf Bundesebene wird eine Vollerhebung der Bevölkerungszahl und -struktur bestenfalls alle zehn Jahre im Rahmen einer Volkszählung (Zensus) durchgeführt. Dieser Rhythmus entspricht den internationalen Minimalvorgaben für Zensen (vgl. z. B. Grohmann 2009; Wagner 2010). Aufgrund der Wiedervereinigung und von konzeptionellen Problemen liegen die letzten Zensen in Deutschland allerdings erheblich länger als zehn Jahre zurück: In der DDR wurde die letzte Volkszählung 1981, in Westdeutschland 1987 durchgeführt. Der aktuelle Zensus ist bereits angelaufen und wird (mit Stichtag 9. Mai 2011 für die Feststellung der Bevölkerungszahl) Ende 2011 abgeschlossen (s. z. B. http://www.zensus2011.de/).

Nur Vollerhebungen ermöglichen die Ermittlung der Bevölkerungszahl und des Gebäudebestandes auf kleinräumiger Ebene, da Stichproben dafür zu kleine Fallzahlen aufweisen. Selbst der Mikrozensus, eine im internationalen Vergleich verhältnismäßig große Stichprobe, umfasst lediglich ein Prozent der Bevölkerung. Diese Fallzahl ist für aussagekräftige kleinräumige Ergebnisse viel zu klein. Die Einwohnermelderegister, die konzeptionell alle Einwohner erfassen, sind insbesondere in Großstädten fehlerbehaftet, da es kein zentrales Einwohnermelderegister für Deutschland gibt und man sich - trotz gesetzlicher Meldepflicht - faktisch weder ab- noch anmelden muss, um in einer Stadt zu leben. So zeigen die Ergebnisse des Zensustests, dass insbesondere die sogenannten „Karteileichen“ mit der Gemeindegröße zunehmen, in Großstädten über 800.000 Einwohnern liegen sie bei ca. acht Prozent (Statistische Ämter des Bundes und der Länder 2004). Zusätzlich gibt es Bevölkerungsgruppen, die sich nicht anmelden können, da sie illegal in einer Gemeinde bzw. (Groß)Stadt leben.

Gegenwärtig wird davon ausgegangen, dass die Bevölkerungszahl für ganz Deutschland um mehr als 1,3 Mio. Menschen überschätzt wird (Statistisches Bundesamt Deutschland 2008). Aber nicht nur auf gesamtstaatlicher Ebene ergibt sich eine Divergenz zwischen Aufzeichnungen und Wirklichkeit. Demographische Prozesse unterscheiden sich räumlich in Rückgang, Alterung und Wanderungsbewegungen der Bevölkerung (Kilper und Müller 2005). So wird für München im Vergleich zu Städten wie Leipzig auch zukünftig ein Bevölkerungswachstum prognostiziert. Diese Prozesse haben entscheidenden Einfluss auf die Siedlungsentwicklung und -dichte, den Flächenverbrauch, die Mobilität sowie die technischen Infrastruktursysteme.

Es stellt offenbar selbst für Länder mit differenzierten Verwaltungsstrukturen und Meldepflicht eine Herausforderung dar, jederzeit aktuelle und zugleich exakte Auskünfte über die Bevölkerungszahl des Landes zu geben. In Ländern mit einer um ein Vielfaches höheren Bevölkerungsdy- namik wird diese Aufgabe zum echten Problem. Wenn man sich die Situation rasant wachsender Megastädte vor Augen führt, kann man erahnen, wie schwierig es ist, einen Überblick über den teilweise unkontrollierten Bevölkerungszuwachs zu erhalten. Viele vor wenigen Jahren noch global unbedeutende Städte in Entwicklungs- und Schwellenländern, insbesondere in Asien, haben sich in den vergangenen drei bis vier Jahrzehnten zu Megastädten mit mehr als zehn Millionen Einwohnern entwickelt bzw. sind auf dem Sprung solche zu werden. Dort werden Menschen mit der Hoffnung auf ein lebenswerteres Leben förmlich in die Städte gespült und sorgen somit für einen kontinuierlichen Zustrom in die Städte. Die Armut drängt viele Menschen dazu, sich auf illegale Weise niederzulassen. Mit der wachsenden Bevölkerung geht ein enormes Flächenwachstum einher. An einer kontrollierten Stadtentwicklung können Städte mit einer solchen Dynamik nur schwer festhalten.

Für einen Außenstehenden wird nirgendwo das Wachstum der Städte so deutlich wie bei einer Betrachtung von oben. Aus der Vogelperspektive erschließt sich dem Betrachter das gesamte Ausmaß der Urbanisierung. Die errechnete Dimension urbaner Flächenexpansion für das genannte Beispiel Kairo zeigt eine Verdreifachung der urbanisierten Flächen seit den 1970er Jahren (Taubenböck et al. 2009; vgl. Abb. 1). Es ist daher naheliegend, dass der Blick von oben Erkenntnisse über aktuelle Anzahl und Verteilung der Bevölkerung in bestimmten Regionen zulässt. Dieser Blick kann mit Hilfe hoch aufgelöster Satellitenfotos nahezu jederzeit aktualisiert werden. Er mag nicht hundertprozentig exakt sein, aber das sind Zensen und andere statistische Erhebungen auch nicht. Und: Wiederholte, gegebenenfalls permanente Blicke sind - vorausgesetzt, dass ein entsprechender Satellit bereits in einer Erdumlaufbahn ist - verhältnismäßig preiswert und datenschutzrechtlich völlig unproblematisch, da einzelne Personen nicht erfasst und identifiziert werden können.

\section{Satelliten zur Volkszählung?}

Inwieweit die räumlichen Daten aus Fernerkundungsmessungen über den eigentlichen Inhalt hinaus auch indirekte Aussagen zulassen, zeigen Sutton et al. (2001). Sie machen sich den Zusammenhang zwischen menschlichem Wirken und Veränderungen auf der Erde zunutze und zeigen, wie Bevölkerungszahlen auf globaler Ebene mit Satellitenbilddaten abgeschätzt werden können. Für ihre Untersuchung benutzten sie nächtliche Satellitenaufnahmen, die die Helligkeit der Erdoberfläche aufgrund künstlichen Lichts als Indikator für die Bevölkerungsdichte von über 1.000 Städten erfassten („census from heaven“). Zusätzliche Informationen können daraus für die wirtschaftliche Leis- 
Abb. 1 Flächenwachstum der Megacity Kairo, abgeleitet aus einer Zeitreihe an Erdbeobachtungsdaten. (Datengrundlage für 1972: Landsat MSS, 1984: Landsat TM, 1990: Landsat TM, 2000: Landsat TM, 2008: TerraSAR-X)

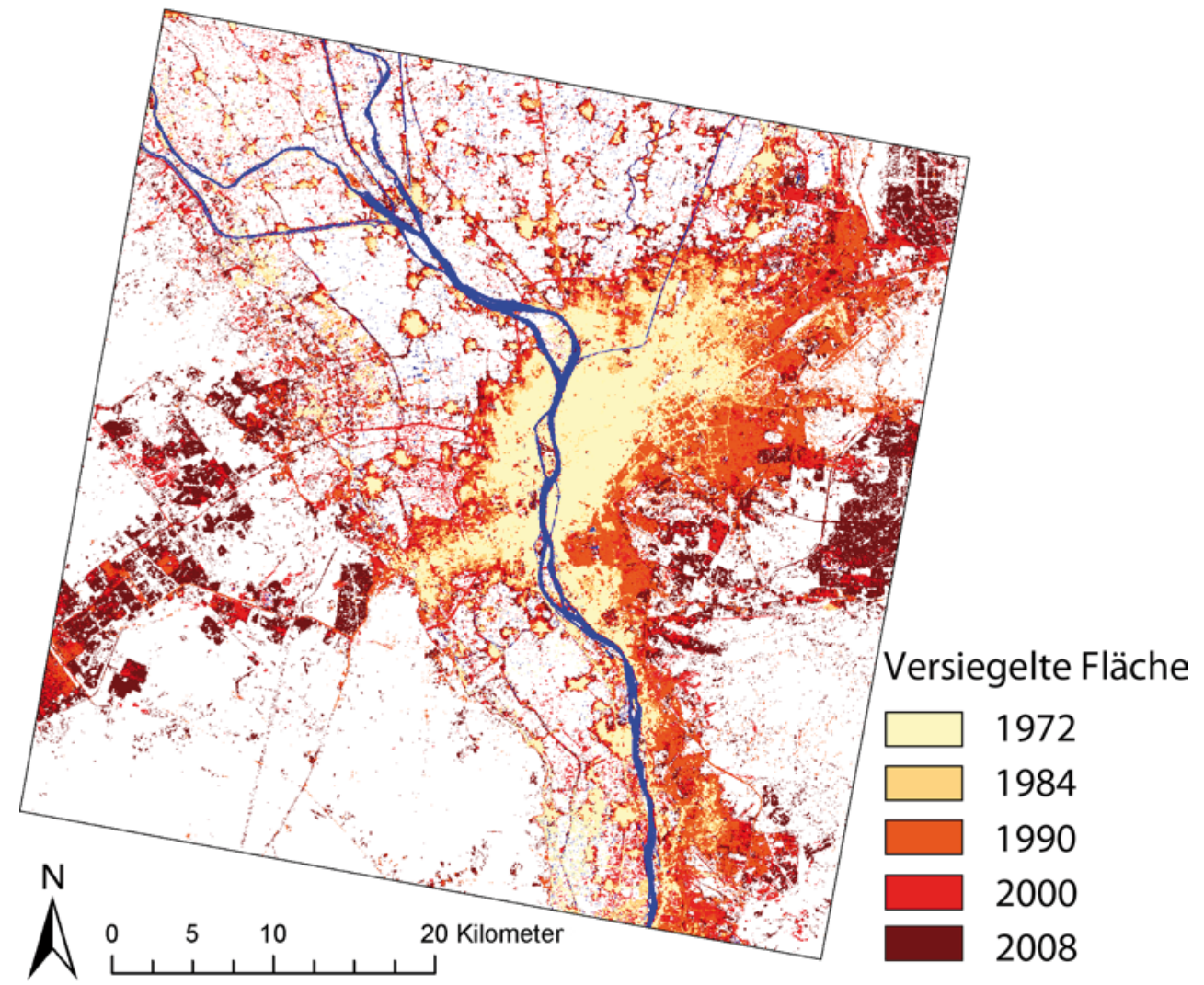

tungsfähigkeit einer Stadt abgeleitet werden, da mit steigendem Wohlstand auch das Ausmaß künstlicher Lichtquellen zunimmt (Henderson et al. 2009). Fienberg und Prewitt (2010) fordern dazu: „The task ahead is formidable. Census communities need to gather ideas for creating more robust data collection using administrative and electronic sources to supplement the [...] surveys and to help address problems of non-response. National census offices need to become more nimble in testing methodologies without jettisoning the best of current approaches."

Für flächendeckende Anwendungen auf regionaler, nationaler oder gar globaler Ebene werden für räumliche Abschätzungen von Bevölkerungszahlen meist Fernerkundungsdaten von Aufnahmesystemen mit großen flächenmäßigen Abdeckungen verwendet. Diese Daten besitzen aber aufgrund ihrer geringeren geometrischen Auflösung nur begrenzte Möglichkeiten, die komplexe Struktur des städtischen Raumes samt seiner vertikalen Komponente zu erfassen. Seit einigen Jahren werden vor allem in Industrieländern vermehrt dreidimensionale Informationen von Städten durch hoch detaillierte Stereoaufnahmen von Satelliten oder durch flugzeuggetragene Laserscanningsysteme gewonnen, weshalb bereits für viele Städte 3D-Stadtmodelle zur Verfügung stehen. Diese können für eine detaillierte Schätzung des Hausbestandes als zwar indirekte, aber jederzeit aktuelle, preiswerte und „nicht-invasive“ Messung der
Bevölkerung herangezogen werden. In der westlichen Welt steht dabei die kleinräumige Verteilung der Bevölkerung im Vordergrund, da für die Gesamtbevölkerung konventionelle statistische Informationen zur Verfügung stehen. In Ländern, in denen die amtliche Statistik teilweise noch großen Aufholbedarf hat, können Fernerkundungsdaten auch für die Abschätzung absoluter Bevölkerungszahlen - etwa von Megastädten - nützlich sein.

Aufgrund umfangreicher Datengrundlagen in entwickelten Ländern - sowohl fernerkundlicher als auch statistischer Natur - ist es möglich, Methoden für die Schätzung der Bevölkerung in weniger entwickelten, hoch dynamischen Städten und Ländern auszuarbeiten und zu testen. Auf diese Weise wird Wissen und Erfahrung gesammelt, was für eine Anwendung der Methode in Gebieten mit geringerer und weniger aktueller Informationsgrundlage hilfreich ist.

München ist nicht Mumbai. Um Möglichkeiten der Fernerkundung zur Unterstützung bei der Lösung elementarer humanitärer Probleme in Schwellen- und Entwicklungsländern auszuloten, ist es jedoch ein guter Ausgangspunkt. Mit Satellitenbildern können Einzelgebäude identifiziert werden, über physische Parameter wie Anzahl, Größe oder Höhe der Gebäude kann annäherungsweise auf die Bevölkerungszahl geschlossen werden. 


\section{Wie viele Menschen wohnen wo? Digitale Bildanalyse am Beispiel Münchens}

Wie die Bevölkerungsverteilung in einem urbanen Raum mittels Erdbeobachtung abgeschätzt werden kann, wird exemplarisch am Beispiel Münchens dargestellt. Die bayerische Landeshauptstadt ist heute mit ca. 1,36 Mio. Einwohnern hinter Berlin und Hamburg die drittgrößte Stadt Deutschlands. Als Datengrundlage dienen im vorliegenden Fall detaillierte Satellitendaten und dreidimensionale Informationen aus stereoskopischen Luftbilddaten. Anhand dieser Bilder (vgl. Abb. 2, oben) ist die physische Ausstattung des städtischen Raumes mit einem Detail bis zu einem Meter erkennbar. Optische Satellitenbilddaten in dieser hohen Auflösung gibt es seit 1999.

Den zweiten, für die Ableitung der Höhe der einzelnen Gebäude relevanten Datensatz bildet das digitale Oberflächenmodell (DOM, vgl. Abb. 2 unten). Dieses wird von einem Kamerasystem erstellt, das auf einem Flugzeug angebracht ist. Das System HRSC-AX (High Resolution Stereo Camera - Airborne Extended) wurde ursprünglich für die russische „Mars 96 Mission“ vom Deutschen Zentrum für Luft- und Raumfahrt (DLR) entwickelt. Bei der Befliegung befindet sich neben den optischen Aufnahmeinstrumenten zusätzlich ein Positionierungssystem an Bord, welches die exakte Verortung des Flugzeugs und der Aufnahmen ermöglicht. Aus den einzelnen stereoskopischen Bilddaten wird mittels photogrammetrischer Verfahren ein Oberflächenmodell in gleicher geometrischer Auflösung wie das Satellitenbild hergestellt.

Die Auswertung der beiden Datensätze erfolgt mittels weitgehend automatisierbarer Bildauswerteverfahren, welche die Objekte nach bestimmten Merkmalen einer thematischen Klasse zuweisen. Basierend auf dem DOM können somit die erhöhten künstlichen Objekte der Landschaft - sprich Gebäude - zu räumlichen Einheiten zusammengefasst werden und von den anderen Elementen unterschieden werden. Zusätzlich kann die Spektralinformation der optischen Satellitendaten zur Ableitung weiterer stadträumlicher Elemente auf der Landoberfläche verwendet werden. So werden nicht nur Gebäude oder Straßen abgeleitet, sondern auch versiegelte Bereiche, Vegetationsflächen, offener Boden oder Wasserflächen klassifiziert.

Die Kenntnis über die räumliche Ausdehnung des Siedlungsraumes - aus Erdbeobachtungsdaten erhoben - ist Grundlage für die Abschätzung der Bevölkerungsverteilung. Über zwei Ansätze können daraus räumliche Verteilungen der Bevölkerung abgeschätzt werden. Während beim „bottom-up-Ansatz“ für den erhobenen Wohnraum eine potenzielle Anzahl wohnhafter Bevölkerung angenommen wird, wird bei der ,top-down-Methode“ eine bekannte (oder geschätzte) Anzahl der Gesamtbevölkerung über die einzelnen Wohneinheiten verteilt. Im vorliegenden Fall werden bekannte Bevölkerungszahlen aus administrativen

Abb. 2 Satellitenbildaufnahme (oben) und Oberflächenmodell (unten) vom Zentrum Münchens. (Quelle: IKONOS: 07/2005 [C EUSI], HRSC-AX: 09/2004; DLR 2011)

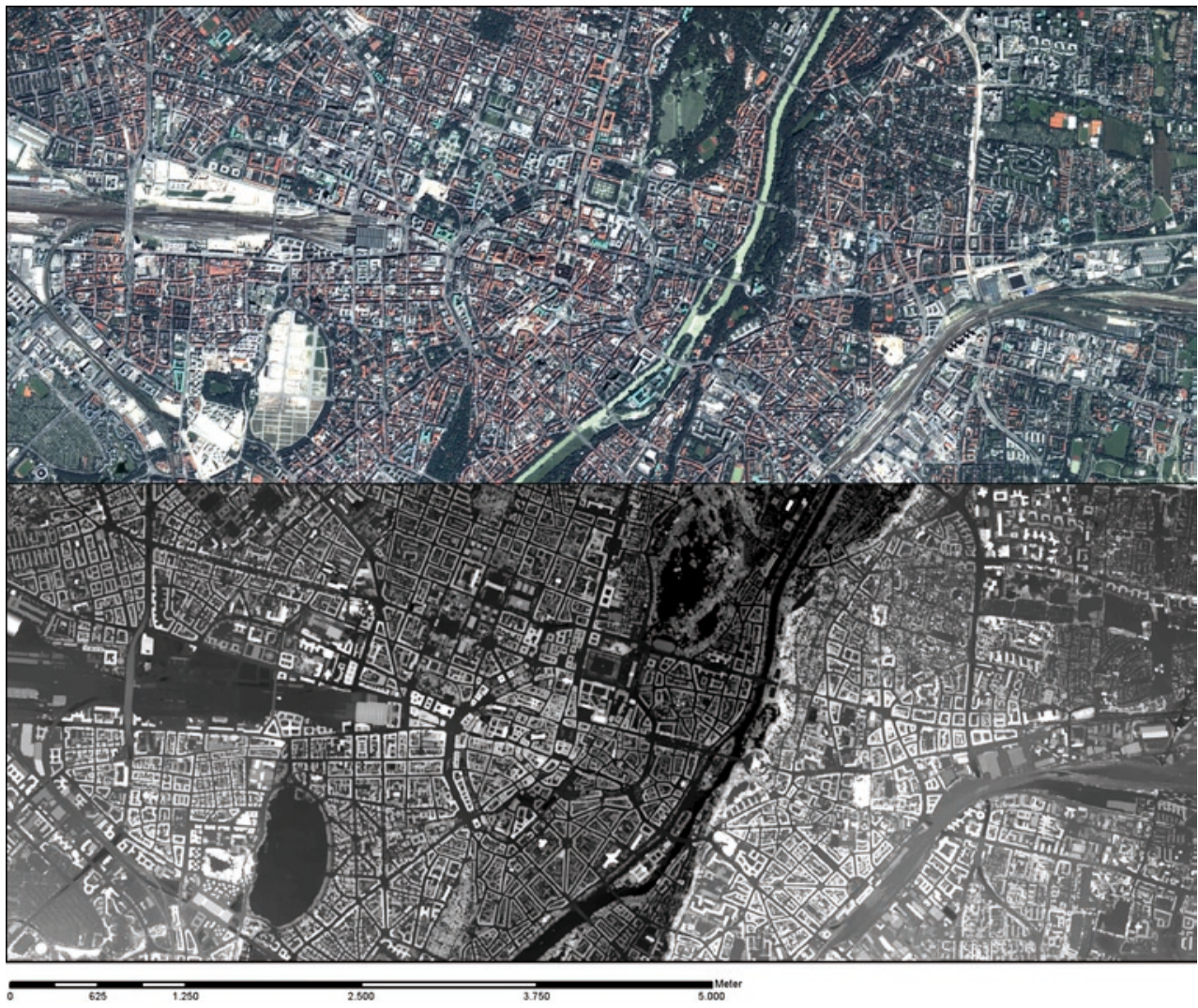


Abb. 3 Bevölkerungsanzahl für die Stadtbezirke (links) und die Stadtbezirksteile (rechts) von München. (Quelle: DLR)

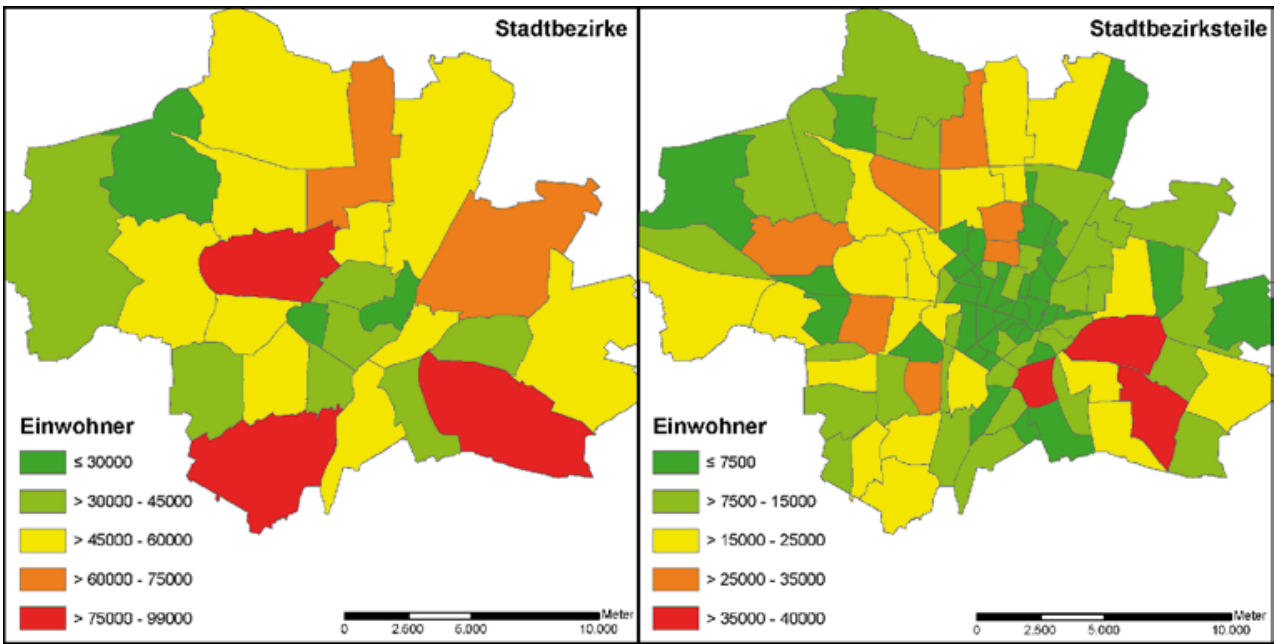

Raumeinheiten der Stadt München auf das 3D-Stadtmodell verteilt. Aus methodischen Gründen wird im Folgenden angenommen, dass die kleinräumigen Daten zur Bevölkerungsgröße, die die amtliche Statistik ermittelt, stimmen. Der Ansatz für die räumliche Disaggregation (Steinnocher et al. 2005) basiert auf Münchner Bevölkerungsdaten, die auf der Ebene von Stadtbezirken bzw. Stadtbezirksteilen zur Verfügung stehen. Die Methode zielt auf eine Verteilung der Bevölkerungsdaten auf die räumlichen Einheiten der Einzelgebäude ab, welche im 3D-Stadtmodell repräsentiert sind. Wie aus Abb. 3 hervorgeht, zeigt sich: Je feiner die Bevölkerungsdaten bereits aufgelöst sind, desto stärker können lokale Unterschiede in der tatsächlichen Bevölkerungsverteilung berücksichtigt werden.

Nicht alle Gebäude einer Stadt werden für Wohnzwecke genutzt, weshalb für eine Bewertung der lokalen Bevölkerungsverteilung Nutzungsinformationen zu den Gebäuden relevant sind. Die Gebäudenutzung kann allerdings nicht direkt aus Fernerkundungsdaten erhoben werden, bestimmte physische Strukturmerkmale können jedoch Hinweise auf die Gebäudenutzung geben. So kann beispielsweise angenommen werden, dass flächenmäßig besonders große Gebäudekomplexe nicht zu Wohnzwecken genutzt werden. Auf der Basis von Formkriterien kann daher eine grobe Differenzierung der Nutzung (Wohnnutzung/Nicht-Wohnnutzung) vorgenommen werden. Zusätzliches Wissen kann über weitere flächenhafte Nutzungsdaten wie ATKIS (Amtliches Topographisch-kartographisches Informationssystem) oder den Bearbeiter eingebracht werden.

Das Potenzial, das darin steckt, aus fernerkundlichen Datensätzen extrahierte 3D-Stadtmodelle als physische Grundlage zur räumlichen Verfeinerung von Bevölkerungsdaten zu nutzen, wird bei einer Gegenüberstellung der Abb. 3 und 4 deutlich. Somit stehen kleinräumige Bevölkerungsdaten zur Verfügung, die nicht durch eine Aggregation auf administrative künstliche Raumeinheiten lokale Konzentrationen verschleiern. Abbildung 4 zeigt exemplarisch die Bevölkerungsverteilung für einen Ausschnitt südöstlich des Münchner Stadtzentrums.

Abgleiche zwischen der ermittelten Bevölkerungsabschätzung und den Referenzdaten zeigen, dass vor allem für Stadtbezirksteile mit hohem Wohnnutzungsanteil (Am alten
Abb. 4 Ergebnis der Bevölkerungsverteilung auf Gebäudeebene für einen Ausschnitt südöstlich des Münchner Stadtzentrums. (Quelle: Wurm und Taubenböck 2010)

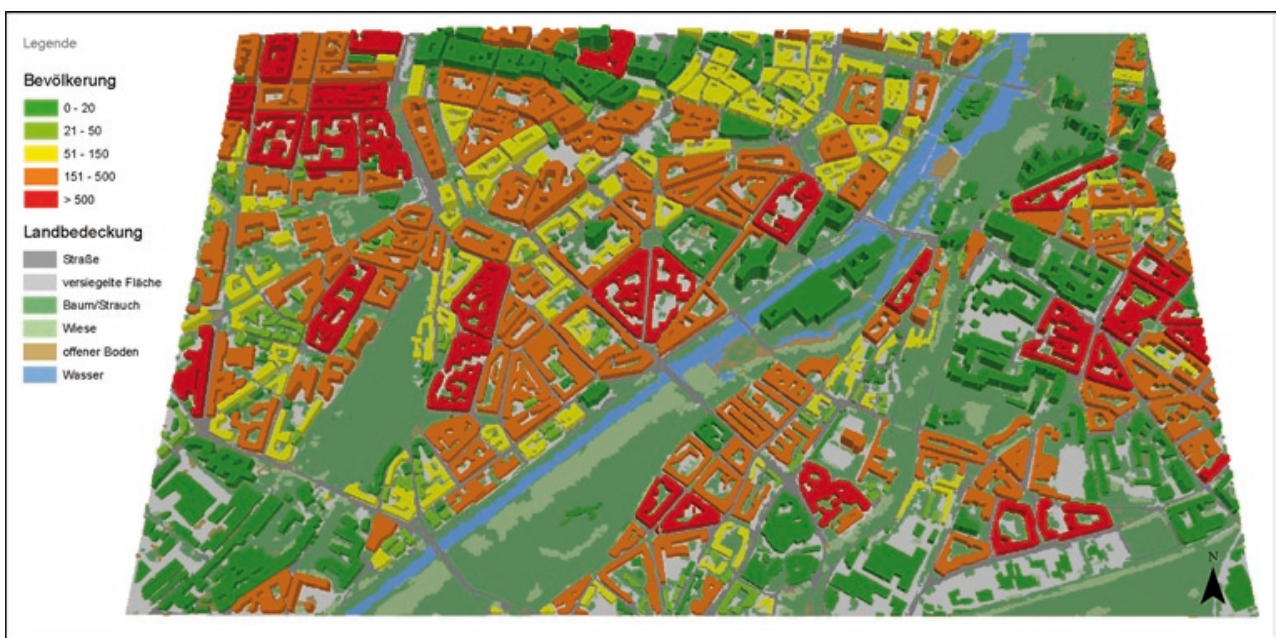


südlichen Friedhof, Haidhausen-Nord und -Süd, Untere $\mathrm{Au}$, Sendlinger Feld, Mittersendling, Westend, St. Vinzenz, Dom Pedro, Untergiesing, Friedenheim und St. Ulrich) hohe Genauigkeiten der Bevölkerungsverteilung mit Abweichungen von weniger als $\pm 5 \%$ erzielt werden. In Gebieten mit einer höheren Durchmischung der Gebäudenutzung bzw. sogar einem überwiegenden Anteil gewerblich und industriell genutzter Gebäude, zeigen sich aber auch Limitierungen dieses Ansatzes. Besonders schlägt sich dies in den Stadtbezirksteilen Kreuzviertel, Ludwigsvorstadt-Kliniken und Königsplatz nieder, welche neben der Hauptgeschäftsstraße Münchens auch universitäre Einrichtungen und Kliniken beherbergen.

\section{Erdbeobachtung als Mosaiksteinchen zum Zensus der Zukunft?}

Die in diesem Beitrag durchgeführten exemplarischen Berechnungen haben gezeigt, welche Möglichkeiten in der kleinräumigen Abschätzung von Bevölkerungsverteilungen liegen: Für Großstädte können damit Bevölkerungszahlen, die auf den Melderegistern beruhen, in den Raum übertragen werden. Für Megacities unbekannter Größe erlauben sie es, die Einwohnerzahlen abzuschätzen. Für nationale oder gar globale Anwendungen, die einen modernen Zensus unterstützen könnten, stehen bereits heute flächendeckende Satellitenbilddaten - allerdings mit einer noch zu groben Auflösung - zur Verfügung. Diese könnten als Mosaiksteinchen eingesetzt werden, um konventionelle Befragungen zu unterstützen. Die Genauigkeiten sind vielversprechend - insbesondere für Gebiete mit überwiegender Wohnnutzung. Sie zeigen aber auch die Limitierungen, welche durch zusätzliche Daten (beispielsweise zu den Gebäudenutzungen) reduziert werden müssen.

Mit punktuellem exaktem Wissen über Bevölkerungszahlen in bestimmten Gebäudetypen besteht auch die Möglichkeit, diese Informationen über Extrapolationsmethoden auf ganze Stadtgebiete hochzurechnen. Mit solchen Hochrechnungen ließen sich geschätzte und statistische Bevölkerungszahlen von Städten erfassen und bewerten. Gerade für das eingangs angeführte Beispiel der Megastadt Kairo verspricht dieser Ansatz, die enormen Unsicherheiten bezüglich der absoluten Bevölkerung zu reduzieren.

Zusätzlich gilt: Würden aufgrund eines ,permanenten Zensus" nach schweizerischem Vorbild gute Bevölkerungsdaten auf kleinräumiger Ebene vorliegen, könnten mit Erdbeobachtungsdaten zusätzliche Merkmale für räumliche Analysen erhoben werden - um beispielsweise die Nachbarschaft der Bevölkerung zu charakterisieren (etwa Gebäudetypen) oder Informationen über sich verändernde Umweltbedingungen (etwa Luftbelastungen) und ihren Einfluss auf die lokalen Lebensbedingungen zu untersuchen.
Es bleibt jedoch zu betonen, dass die hier vorgestellte Methode einen Zensus keinesfalls ersetzen kann. Die Ergebnisse der Fernerkundung können aber dazu dienen, Zensusdaten noch aussagekräftiger zu machen, als sie es alleine sein können. Angesichts des Aufwandes, der mit einem Zensus verbunden ist, sollten alle Möglichkeiten geprüft werden, die Aussagekraft der konventionellen Zensusdaten im oben beschriebenen Sinne von Fienberg und Prewitt (2010) zu erhöhen. Diese Möglichkeiten konnten hier nicht erschöpfend diskutiert werden. Der vorliegende Aufsatz kann und will nicht Zensen ihre Legitimation absprechen. Stattdessen will er zur Diskussion anregen, inwiefern moderne und etablierte Technologien Zensen unterstützen - zu einem ,census from heaven“ (Sutton et al. 2001).

\section{Literatur}

Bradburn NM (1993) A census that mirrors America. National Research Council, Washington

Bundesministerium der Justiz (2009) Bevölkerungsstatistikgesetz (BevStatG, BGBI. I S. 1290) und Bundesstatistikgesetz (BStatG, BGB1. I S 2246). http://bundesrecht.juris.de

Fienberg S, Prewitt K (2010) Save your census. Nature 466:1043

Grohmann H (2009) Von der Volkszählung zum Registerzensus: Paradigmenwechsel in der deutschen amtlichen Statistik. AStA Wirtschafts- und Sozialstatistisches Archiv 3:3-23

Henderson JV, Storevgard A, Weil DN (2009) Measuring Economic Growth from Outer Space. NBER Working Paper No. 15199. Cambridge, Mass

Kilper H, Müller B (2005) Demographischer Wandel in Deutschland. Geogr Rundsch 3:36-41

Landeshauptstadt München (2009) Statistisches Amt der Stadt München. http://www.muenchen.de/Rathaus/dir/statistik/37879/ index.html

Statistische Ämter des Bundes und der Länder (2004) Ergebnisse des Zensustests. Wirtsch Stat 8:813-833

Statistisches Amt der Landeshauptstadt München (2006) Statistisches Taschenbuch 2006. München und seine Stadtbezirke. S 195. http://www.mstatistik-muenchen.de/themen/stadtbezirke/stadtbezirkszahlen 2005/taschenbuch 2006.pdf

Statistisches Bundesamt Deutschland (2008) Pressemitteilung Nr. 265 vom 22.07.2008. http:/www.destatis.de/jetspeed/portal/cms/ Sites/destatis/Internet/DE/Presse/pm/2008/07/PD08_265 121 ,templateId $=$ renderPrint.psml

Steinnocher K, Petrini F, Tötzer T, Weichselbaum J (2005) Räumliche Disaggregation von sozio-ökonomischen Daten, Angewandte Geographische Informationsverarbeitung XVII-Beiträge zum AGIT-Symposium

Sutton P, Roberts D, Elvidge C, Baugh K (2001) Census from Heaven: an estimate of the global human population using night-time satellite imagery. Int J Remote Sens 22(16):3061-3076

Taubenböck H, Wegmann M, Roth A, Mehl H, Dech S (2009) Analysis of urban sprawl at mega city Cairo, Egypt using multisensoral remote sensing data, landscape metrics and gradient analysis. Proceedings of the ISRSE conference, Stresa, Italy. S 4

Wagner GG (2010) Volkszählung bitte nicht überfrachten - vor allem nicht hinsichtlich der Erwartungen. RatSWD Working Paper No. 134. Berlin 
Wurm M, Taubenböck H (2010) Abschätzung der Bevölkerungsverteilung mit Methoden der Fernerkundung. In: Taubenböck H, Dech S (Hrsg): Fernerkundung im urbanen Raum - Erdbeobachtung auf dem Weg zur Planungspraxis. Wissenschaftliche Buchgesellschaft, Darmstadt, S 143-152

Mag. rer. nat Michael Wurm, Jg. 1980. Studium der Geographie an der Karl-Franzens-Universität Graz. 2008-2011 wissenschaftlicher Mitarbeiter am Lehrstuhl für Fernerkundung der Bayerischen JuliusMaximilians-Universität Würzburg. Seit 2011 wissenschaftlicher Mitarbeiter am Deutschen Fernerkundungsdatenzentrum (DFD) im Deutschen Zentrum für Luft- und Raumfahrt (DLR) in Oberpfaffenhofen. Arbeitsschwerpunkte: Fernerkundung urbaner Räume, Synergien von Fernerkundung und sozialwissenschaftlichen Daten.

Dr. Hannes Taubenböck, Jg. 1977. Studium der Geographie an der Ludwigs-Maximilians-Universität München. Doktorand am DLRDFD und Promotion an der Bayerischen Julius-Maximilians-Universität Würzburg 2008. 2007-2010 wissenschaftlicher Mitarbeiter am Lehrstuhl für Fernerkundung der Universität Würzburg. Seit 2009 wissenschaftlicher Mitarbeiter am Deutschen Fernerkundungsdatenzentrum (DFD) im Deutschen Zentrum für Luft- und Raumfahrt (DLR) in Oberpfaffenhofen. Arbeitsschwerpunkte: Fernerkundung urbaner Räume, Vulnerabilität und interdisziplinäre Studien.

Dr. Jan Goebel, Jg. 1971. Studium der Soziologie und Volkswirtschaft an der Technischen Universität Berlin. Promotion 2006 an der TU Berlin. Seit 2001 wissenschaftlicher Mitarbeiter in der forschungsbasierten Infrastruktureinrichtung Sozio-oekonomisches Panel (SOEP) am Deutschen Institut für Wirtschaftsforschung Berlin (DIW Berlin). Seit 2010 stellvertretender Leiter des Forschungsdatenzentrums SOEP. Arbeitsschwerpunkte: Einkommensverteilung, Item-Nonresponse, Regionalindikatoren.
Dr. Thomas Esch, Jg. 1975. Studium der angewandten physischen Geographie an der Universität Trier, Doktorand am DLR-DFD und Promotion an der Bayerischen Julius-Maximilians-Universität Würzburg 2006. 2006-2008 wissenschaftlicher Mitarbeiter am Lehrstuhl für Fernerkundung der Universität Würzburg. Seit 2008 wissenschaftlicher Mitarbeiter am DLR-DFD und seit 2009 Leiter der Arbeitsgruppe „Siedlungsraum und Landmanagement“. Arbeitsschwerpunkte: Fernerkundung urbaner Räume, Anwendungs- und Methodenentwicklung für SAR-Daten.

Prof. Dr. Gert G. Wagner, Jg. 1953. Studium der Volkswirtschaft an der Johann Wolfgang Goethe-Universität Frankfurt, Promotion 1984 an der TU Berlin, Habilitation 1992 an der TU Berlin. Seit 1989 Leiter der Längsschnittstudie Sozio-oekonomisches Panel am DIW Berlin, seit 2002 Professor für Volkswirtschaftslehre an der TU Berlin. Seit 2008 Max Planck Fellow am MPI für Bildungsforschung Berlin und seit 2010 Vorsitzender des Vorstands des Deutschen Instituts für Wirtschaftsforschung (DIW Berlin). Leiter der wissenschaftlichen Kommission zur Begleitung des Zensus 2011 („Zensuskommission“). Arbeitsschwerpunkte: Politikberatung, verhaltens- und arbeitsmarktpolitische Analysen, Surveystatistik, Universitätslehre VWL. 